\title{
Representation of the third dimension: The use of perspective cues by 3- and 4-month-old infants
}

\author{
Karine Durand $^{\mathrm{a}}$, Roger Lécuyer ${ }^{\mathrm{b}, *}$, Myriam Frichtel $^{\mathrm{b}}$ \\ ${ }^{\text {a }}$ LEAD Université de Dijon/CNRS, Dijon, France \\ ${ }^{\mathrm{b}}$ Laboratoire Cognition et Développement, Université René Descartes/CNRS, \\ 71 Avenue Edouard Vaillant, 92774 Boulogne-Billancourt Cedex, France
}

Received 30 May 2001; received in revised form 18 October 2002; accepted 14 November 2002

\begin{abstract}
In recent years, the old debate about the beginning of representational capacities in childhood has been revived, fueled by new evidence of early cognitive capacities. This new evidence necessitates further reflection on the nature of the representations involved in early cognitive functioning. One important goal is to determine which situation will provide a better understanding of these representations. We hypothesize that the use of depth cues, especially perspective cues, in a two-dimensional (2-D) display is one of these situations, because the perceiver needs to build a representation from incomplete information. Three experiments were carried out to test the capacity of young infants to use perspective cues to build a three-dimensional (3-D) representation in a 2-D display. In the test phase of the first experiment, infants did not evidence a capacity to extrapolate to apparently further objects a size/apparent distance relation presented to them in the habituation phase. However, in the second experiment, infants dishabituated with apparently nearer objects when this rule was violated. In the third experiment, infants also dishabituated to a situation where an object was apparently growing in size when moving away and decreasing when coming nearer. These results seem to indicate that this representational capacity emerges between the age of 3 and 4 months. These results are discussed in the context of the nativist/empiricist debate. (C) 2003 Elsevier Science Inc. All rights reserved.
\end{abstract}

Keywords: Infant; Representation; Perspective; 2-D/3-D

${ }^{*}$ Corresponding author. Tel.: +33-1-55-59-90.

E-mail addresses: kdurand@u-bourgogne.fr (K. Durand), roger.lecuyer@psycho.univ-paris5.fr (R. Lécuyer). 


\section{The question of representation in infants}

The classical conceptions of representation and representational capacities have focused on symbolic representation. In the philosophical tradition this kind of representation had been thoroughly linked to language. Many psychologists (e.g., Wallon, 1942) carried on this tradition and for a long time, the idea of representation before language acquisition was completely excluded. Piaget (1936) proposed another criterion as evidence of earlier representational capacity: object permanence. Debates about early representation focused first on this representational level and the necessity (or nonnecessity) of language mastery as a condition for representation. From that time on the debate about representation was also a debate about the forms of representations.

However, while Piaget's theory was the most popular theory in developmental psychology, object permanence was the main criterion used as the evidence of representational capacity.

Over the last 20 years of research in cognitive development in infants, many early capacities have been demonstrated, and the thesis of a close relation between representation and language has been questioned. Moreover, the current debate about early object permanence (Haith, 1998; Spelke, 1998) is also a debate about representation (Meltzoff \& Moore, 1998). Long before the question of representation in infants had been raised on a theoretical ground, it had been posed by a lot of new experimental results such as neonatal imitation (Meltzoff \& Moore, 1977), object permanence (Baillargeon, Spelke, \& Wasserman, 1985), categorization (Fagan, 1976), intermodal transfer (Streri \& Pêcheux, 1986), and even habituation and novelty reaction (Sokolov, 1963).

However, the thesis for the existence of early representations has given rise to great controversy (Haith, 1998). One of the major reasons for this controversy appears to be the extensive confusion surrounding the notion of representation. This notion is very often considered in an all or nothing way of thinking and different levels or types of representation are rarely taken into account.

In a recent review about representation, Mandler (1998) made the distinction between procedural and conceptual representation. She also compared the other classical dichotomies, such as accessible vs. inaccessible, conscious vs. nonconscious and explicit vs. implicit. As she focused her review on infant representation most of these dichotomies seemed to her inadequate (and, for the same reason, "declarative" as opposed to "procedural" knowledge). Mandler also pointed to the existence of a fundamental bias in thought about infants' representation. Since adult representations are closely linked to language, it is very difficult to think about a nonlinguistic representation.

Mandler presented Piaget's theory and his distinction between early sensorimotor (procedural) representation and later conceptual representation. She formulated two major criticisms: an empirical criticism and a theoretical one. The empirical criticism consisted of highlighting that, in the recent years, a series of facts were evidenced that suppose an early conceptual representation (e.g., Baillargeon, 1986). The theoretical criticism advanced that Piaget $(1936,1937)$ did not describe the passage from sensorimotor to conceptual representation in a unique and unequivocal way. Then, she exposed more recent theories of representation (Karmiloff-Smith, 1992; Leslie, 1994; Mandler, 1988, 1992) with the same thread. Finally, she reviewed the types of information infants represent (i.e., representation of objects, perceptual categories and 
concept formation or prelinguistic meanings), while, once again contrasting procedural and conceptual knowledge.

Whatever the importance of this distinction, in children and adults, and the convincing empirical and theoretical arguments for it put forward by Mandler, it does not solve the representational problem in infants: If, as Mandler suggested, conceptual representations exist early in infancy and are not simply perceptual procedural representations, different levels of representation must be distinguished. In fact, Mandler's point of view is weakened by the fact that she assimilates perceptual representation to a procedural representation. In habituation/dishabituation situations, a representation of the first stimulus or event presented is necessarily stored, this storage is the result of the perceptual/memory process but the content of the representation is at least as stimulus-dependent as procedure-dependent and therefore the representation cannot be only defined by the procedure. This does not mean that the neonates' representational system is a symbolic system.

In the same way, Meltzoff and Moore (1998) proposed a model in which newborns have a representational system which is the basis for future cognitive development. As a matter of fact, it seems necessary to assume that early cognitive development is based on a representational system which becomes more complex during infancy. Since Meltzoff and Moore hypothesize there exists a representational capacity at birth, they may be supposed to belong to the nativist trend. However, the representational capacity they hypothesize is limited and does not imply object permanence or knowledge about object identity; it is simply the necessary condition for habituation and novelty reaction. At this first level, a representation appears to be a translation of the stimulus represented within a new coding system. There is a relation of homology between the represented object and its representation. In fact, when a newborn shows evidence of a novelty reaction, she or he necessarily compares the new stimulus to the representation of the familiar one which is necessarily stored in memory.

Concerning the simplest and the more complex representational levels, there is no major problem with regards to the cognitive capacities they necessitate. However, for the intermediate levels, the situation is very different. Meltzoff and Moore (1998) distinguish two types of identities: feature or qualitative identity ( $x$ is the same as $y$ ) and numerical identity ( $x$ is the same). The object is the same whatever its displacements. This supposes that the subject has a memory of the different visible and nonvisible displacements of the object. To illustrate the first type of identity, Meltzoff and Moore refer to research about categorization in infants. However, a categorization capacity supposes a more complex identity criterion than they hypothesize. This criterion may be expressed as: " $x$ will be considered as the same as $y$, in spite of the fact that $x$ differs from $y$ ": Every member of the category must be discriminated and related to others. This level of identity seems very close to what the authors call numerical identity. When an object is included in a category, it is simultaneously considered as the same as, but not as the same. Furthermore, in a categorization experiment, as in an "object permanence" experiment, infants must take into account properties of objects that are no longer present. Concerning numerical identity as defined by Meltzoff and Moore, if an object which disappears and appears again is considered still the same, how is it possible without permanence? However, object permanence supposes a representational level. 
Thus, we propose to distinguish between three levels of representation:

- Level 1: concrete or analogical representation. A representation is a translated copy of a stimulus. If the same stimulus is presented again, it will be recognized. If another stimulus is presented, it will be differentiated.

- Level 2: abstract representation. A representation may include variations in the stimulus, and a common property is detected. When a new stimulus is presented, a comparison is done between its properties and the representation. When the information given is partial, this comparison includes re-building properties absent from the stimulus but present in the representation. When an object disappears, some of its properties (size, solidity, etc.) can be taken into account.

- Level 3: symbolic representation. The relation between the represented object and the representing entity is arbitrary. Language and counting activity are typical of this level.

Level 3 has often been studied and debated. Level 1 has been questioned, but habituation/dishabituation exist and must be explained. Conversely, habituation is a well-known mechanism in infants and can characterize this first representational level. Level 2 is the most problematic level and is not yet well understood. There do exist different ways to better understand this level. One of these ways refers to categorization situations: In these situations, infants take into account properties that have been presented previously (during the categorization experiment or in everyday life), but which need to be abstracted when most of the categorized objects are absent. Another group of classical situations interpreted in terms of representation capacity is that of object permanence situations, which is particularly interesting when the number of hidden objects exceeds one (Wynn, 1992). Two-dimensional (2-D) representations of three-dimensional (3-D) scenes also require the same type of cognitive activity. The pictorial cues present in the stimulus rarely give complete 3-D information and the subject must rebuild the third dimension to have a 3-D representation of the object. Two-dimensional displays appear to be symmetrical to categorization situations: instead of taking into account properties of absent objects, the perceiver needs to take into account absent properties of objects. Therefore, in both situations, the subject must build a complete representation with an incomplete stimulation.

In the nativism/empiricism debate, the question of representation, and more specifically the question of 3-D perception and 2-D displays has indeed been mentioned (Meltzoff \& Moore, 1998; Spelke, 1998). Spelke wondered about an important question: "Do we learn to see objects by looking at and manipulating objects? Alternatively, do structures for representing 3-D scenes furnished with bounded objects develop independently of perceptual encounters with those scenes and objects?" (p. 192). If, as supposed by Spelke, the correct answer to this question is the second answer, that structure exists independently of experience, depth perception must occur on a 2-D scene containing the suitable depth cues. On the other hand, if learning is necessary, using depth cues will appear later in a 2-D display, which is always a cultural product, than in a 3-D real scene which every environment provides at birth.

This paper has two purposes. The first is to provide new facts about the nature of representations. The second is to provide new arguments for the nativism/empiricism debate. A situation in which objects are presented in a perspective context, with a 2-D display, is used. The perception of 3-D object relations when a 2-D display is used supposes a processing of the stimulus that matches 2-D cues and 3-D representation. 


\section{A classical problem: depth perception}

The use of the visual cliff paradigm has shown that 9-month-old infants can perceive depth (Gibson \& Walk, 1960). Later, Campos, Langer, and Krowitz (1970) evidenced the presence of depth perception in 2-month-olds. With a different paradigm, Fantz (1961), replicated by Fantz and Nevis (1967) demonstrated the capacity of very young infants to discriminate 2-D and 3-D stimuli, and also their preference for 3-D. Yonas and Granrud (1985), Yonas and Owsley (1987), Yonas, Pettersen, and Granrud (1982), and Yonas and Pick (1975) studied most of the different aspects of perception of the third dimension (see, Yonas \& Owsley, 1987, for a review). Yonas' studies focused particularly on infants' sensitivity to the different cues of the third dimension (kinetic, binocular, and pictorial information). These studies demonstrated the development of the capacity to implement these different cues, from birth to the second half of the first year. However, the use of a motor response in most of Yonas et al.'s experiments may have led to an underestimation of the infants' capacity to understand depth indices. It has been observed in the relations between motor activity and other aspects of infant cognition that motor responses are neither the conditions to acquire knowledge nor the relevant indices of this knowledge mastery (Baillargeon et al., 1985; Lécuyer, 1994).

\section{A new question: depth representation}

As previously remarked, the question of depth perception has always been considered from the point of view of preference for 3-D stimuli as opposed to 2-D stimuli. This seems to be the consequence of the old idea that perception is first 2-D (because the retina is flat) and becomes 3-D later (Piaget, 1936). However, the consequences of the early preference for 3-D have not been completely taken into consideration. Two-dimensional stimuli rarely exist in natural situations. Usually, the scenes that can be seen are 3-D and 2-D displays of 3-D scenes are always a cultural product (Hudson, 1960, 1967). As a consequence, visual perception is more probably prepared for 3-D than for 2-D displays whose relation with 3-D represented situations must be learned. In other words, in a culture in which images are omnipresent, one of the first representations to be built by infants is representations of representations.

Yonas and Owsley (1987) were the first authors to study pictorial cues that allow a representation of depth in 2-D displays. Using a manual reaching gesture they did not evidence a capacity to use these cues before 5-7 months. However, using a purely visual situation with moving stimuli and a fixation duration index, Lécuyer and Durand (1998) evidenced a sensibility to dynamic interposition in 3-month-olds. Durand and Lécuyer (2002) also evidenced in 4-month-olds a capacity to understand a cognitively complex situation-a 2-D replication of object permanence situations used by Baillargeon and Graber (1987) and Baillargeon and DeVos (1991)—presented on a TV screen when dynamic perspective and interposition cues were available. Since young infants can use dynamic interposition cues (Lécuyer \& Durand, 1998) and dynamic interposition plus perspective cues (Durand \& Lécuyer, 2002), a question that remains to be answered is whether infants are able to perceive depth in a 2-D display when perspective (static and dynamic) is the only available cue. 


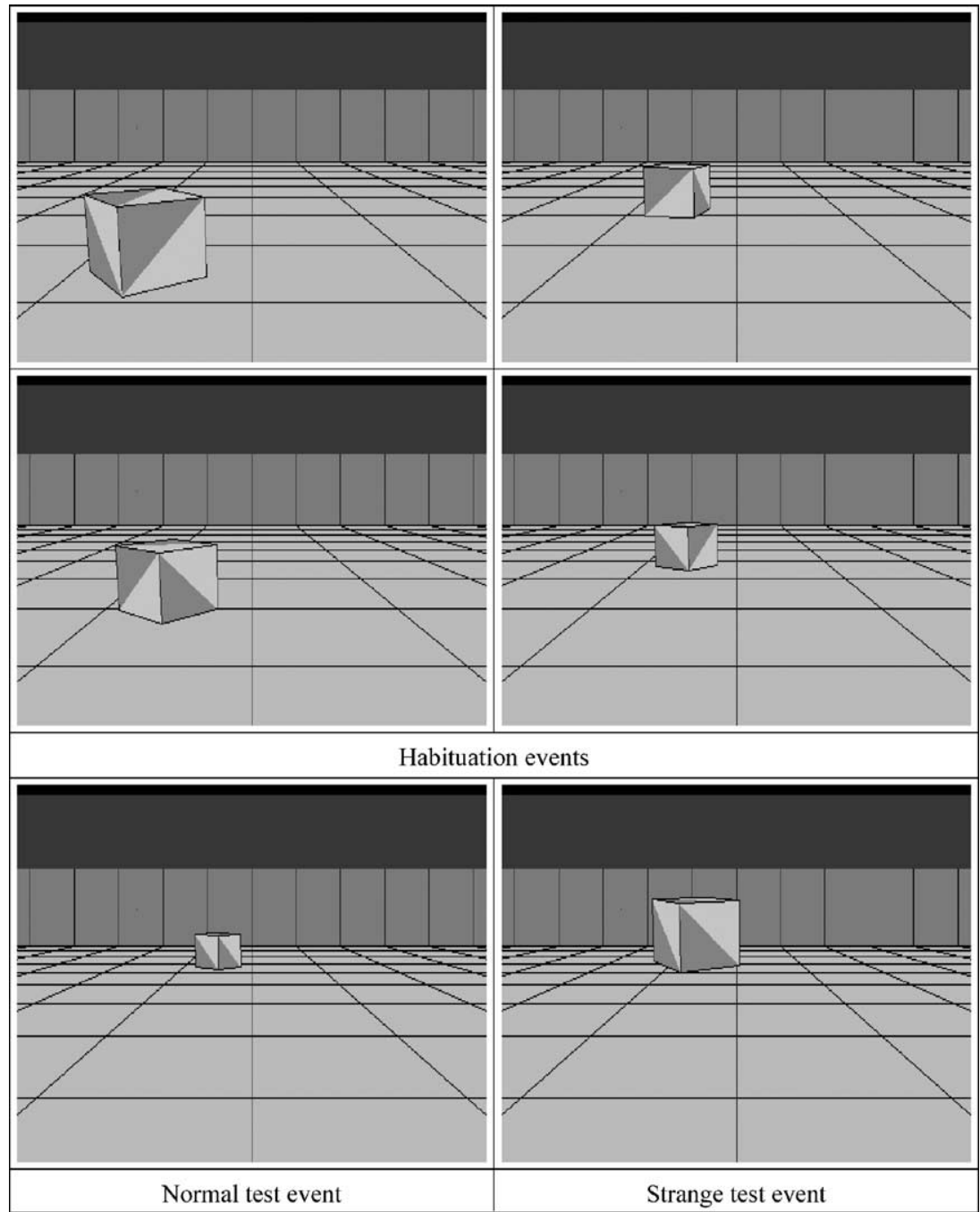

Fig. 1. The habituation events, normal and strange test events; experiment 1.

With a habituation/violation-of-expectation paradigm, three experiments were carried out. Using a 2-D display, an object was presented to infants with a background presenting many perspective cues (see Fig. 1). The object's size was varied as a function of its apparent distance. The common purpose of the three experiments was to test infants' capacity to use these perspective cues. Slater, Mattock, and Brown (1990) demonstrated size constancy in newborns. These results imply that, as early as birth, infants can take into account the relation between size and distance in a 3-D situation. The question now is to determine when they can take into account the same relation in a 2-D display. If the nativist point of view is correct, then 3-month-old infants can use 3-D cues and will look longer at a strange situation in which the relation between distance and size is violated than at a normal situation in which this relation is respected. If the empiricist point of view is true, infants may have the same pattern of responses 
but they also may learn this relation later (in this case, infants will not look longer at the strange situation), or this learning may be under way (in this case, infants' responses will not allow clear cut conclusions).

\section{Experiment 1}

In the first experiment, infants were habituated to a cube displayed at different apparent distances, that is to say what is perceived by adults as different distances, with different sizes related to these apparent distances (see Fig. 1). After habituation, they were confronted with an extrapolation of this rule (the cube was apparently further away and smaller) alternated with a violation of the rule (the cube was apparently further away but its size was the mean size of the habituation stimuli).

\subsection{Subjects}

The experiment was conducted on a sample of 24 full-term 3-month-old infants, 11 girls and 13 boys (mean age $=3$ months, 4 days). Four additional infants were tested but could not be used as subjects because of sleeping or crying. The infants were recruited from the birth registry office in Paris. The parents were contacted by mail and phone and they came to the laboratory where the infants were tested.

\subsection{Apparatus}

A 2-D TV display was used, based on Lécuyer, Humbert, and Findji (1992). The apparatus consisted of a Thomson large screen television ( $85 \mathrm{~cm}$ diagonally) on which subjects observed an animated sequence via a $45^{\circ}$ oriented one-way screen. The infant, seated in front of the one-way mirror, saw only the image of stimuli displayed on the TV monitor. A Panasonic TV camera placed behind the one-way screen, in the axis of the center of the TV image, filmed the infant's looking direction. The camera was connected to a Acorn computer and an experimenter observed the infant on the computer monitor. The experimenter pressed the mouse key when the infant started looking at the video event and stopped pressing when the infant looked away. The experimenter could not see the events presented to infants from her viewpoint and she did not know the order in which the test events were presented. Thus, she was completely blind to the experimental conditions. The video images of the infants were taped for further verification.

In the habituation phase, a trial began by opening a "curtain" on the video screen. Then a cube appeared within a background representing a closed room containing perspective cues. To indicate the volume of the cube, the cube rotated on its central axis and all of its sides were painted yellow and red. The position of the cube in the room was randomly determined by the computer, and the cube was bigger when closer and smaller when further away (Fig. 1). The cube was always on the left side of the room. Thus, its apparent distance was indicated by its $X$ position in the image (apparently near means near the left side of the screen, apparently far means near the center of the screen). Apparent distance was also indicated by the $Y$ position 
(apparently near means near the bottom of the screen, apparently far means near the center of the screen).

A trial began when infants looked longer than one second at the screen and ended when they looked away for more than $1 \mathrm{~s}$ at the end of each trial, a blue curtain was drawn to signal the end of the animated event and the beginning of the next one. The subjects were considered to be habituated when the mean fixation time was less than $50 \%$ of the mean of the first three fixations. As the relation between size and apparent distance was complex, a minimum of nine trials was required before the test phase. Conversely, a maximum of 15 trials was used to avoid infants getting tired.

The test events were presented alternately during 6 trials ( 3 normal events and 3 strange events). The test trials began and ended in the same way as the habituation trials. The normal events were a simple extrapolation of the rule presented in the habituation phase (Fig. 1): the cube displayed was apparently further away and smaller than all the cubes presented in the habituation phase, but the relation between size and apparent distance was the same. The strange events violated this law: the position of the cube was the same as in the normal events, but its size was the mean size of the cubes presented in the habituation phase. Adults looking at these events judged them to be really strange. However, as the size of the cube was familiar, the normal events (in which size and position were new) were newer than the strange events (in which only position was new).

\subsection{Results}

As shown in Fig. 2, no difference appeared in looking durations for the normal and the strange event. Infants looked longer to the strange event but the difference was not significant $[F(1,22)=.29, p=.595]$.

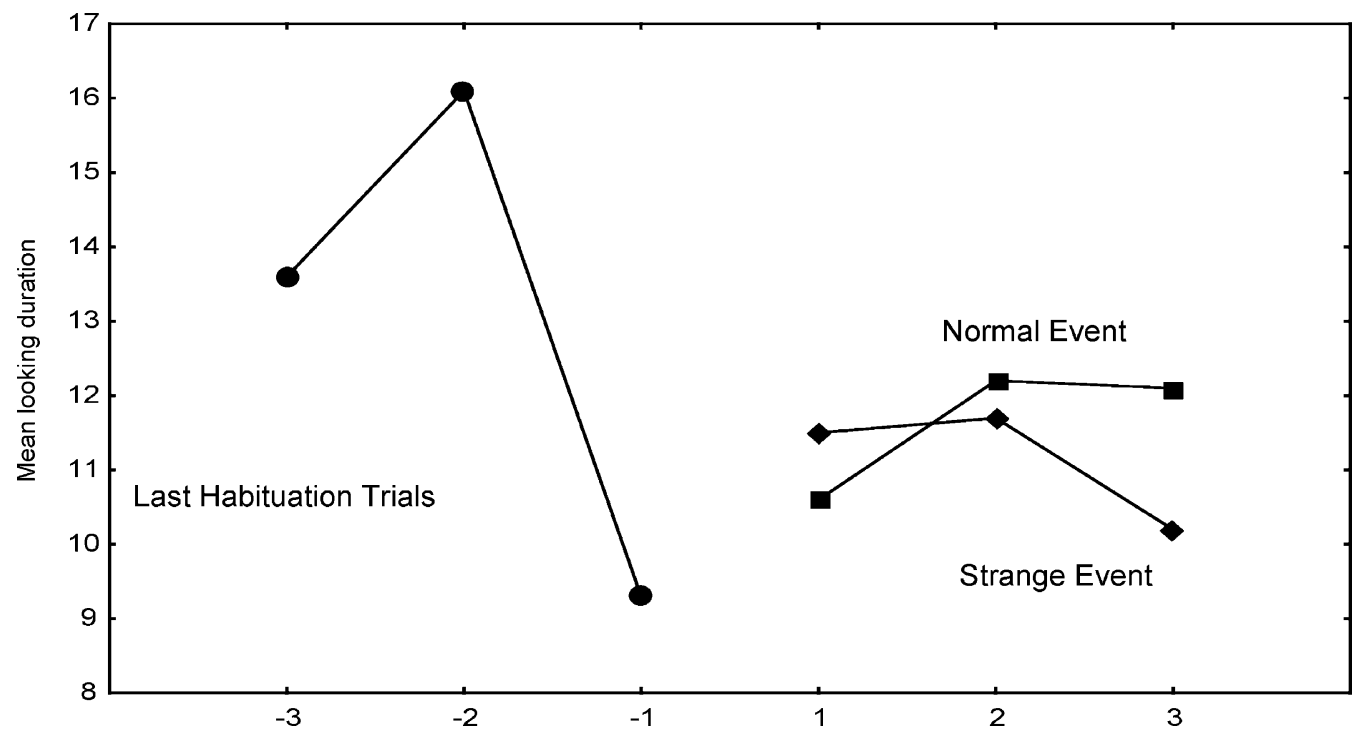

Fig. 2. Mean looking durations in seconds during the last habituation trials, normal test trials and strange test trials; experiment 1. 


\subsection{Discussion}

In the first experiment, no difference was observed between looking duration for the normal and the strange events. Three hypotheses can be considered to explain the absence of difference between looking duration. The first hypothesis is that 3-month-olds are not able to use perspective cues in a 2-D display, as proposed by Yonas and Granrud (1985). A second hypothesis is that a sequential display of the different distances of the object is not adapted to the infants' representation of the relation between distance and size. Three-month-olds should be unable to integrate the successive information given in a unique relation between size and distance. A third hypothesis can also be proposed: infants can understand this physical law, but they cannot extrapolate it when objects move away, maybe because in everyday life, their attention is more focused on approaching objects than on receding objects. A minor change in our first experiment could allow us to test the third hypothesis. Thus, this was the purpose of the second experiment.

\section{Experiment 2}

\subsection{Subjects}

The experiment was conducted on a sample of full-term 3-month-old infants, 11 girls and 13 boys (mean age $=3$ months, 3 days). Five additional infants were tested but could not be used as subjects because of sleeping or crying. The infants were recruited in the same way as in the first experiment.

\subsection{Apparatus and procedure}

The apparatus and procedure used in the second experiment were the same as those used in the first experiment. The habituation phase was exactly the same. In the test phase, two rotating cubes were presented alternately, apparently closer than all the cubes presented in the habituation phase (Fig. 3). The normal event was a big cube presented near, and the strange event was a small cube presented near. The size of the big cube was based on the extrapolation of the rule presented in the habituation phase. The size of the small cube was equal to the mean of the sizes of the cubes presented in the habituation phase.

\subsection{Results}

As shown in Fig. 4, no difference appeared in the looking durations for the normal and the strange events. Infants looked longer at the strange event but the difference was not significant $[F(1,22)=1.291, p=.268]$. Fig. 4 also indicates a significant difference between the last habituation trials and the strange event trials $[F(1,22)=7.477, p=.012]$. Such a significant novelty reaction did not appear for the normal event $[F(1,22)=3.005, p=.092]$. 


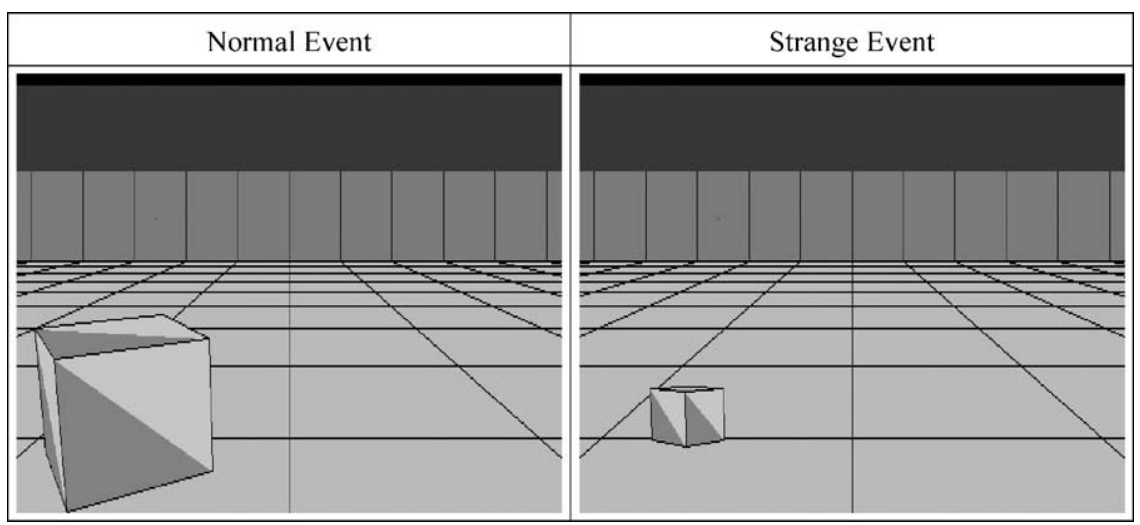

Fig. 3. The habituation events, normal and strange test events; experiment 2.

\subsection{Discussion}

Since no difference was evidenced for the looking durations between the normal and the strange event, our third hypothesis was not supported. However, considering the relations between the last habituation trials and the test trials, a novelty reaction was observed for the strange event, but not for the normal event. This difference indicates that 3-month-olds did not process in the same way the normal and the strange event when the object presented was nearer in the test phase than in the habituation phase. As a consequence, our results appear to be ambiguous because no difference was observed in the looking durations between the

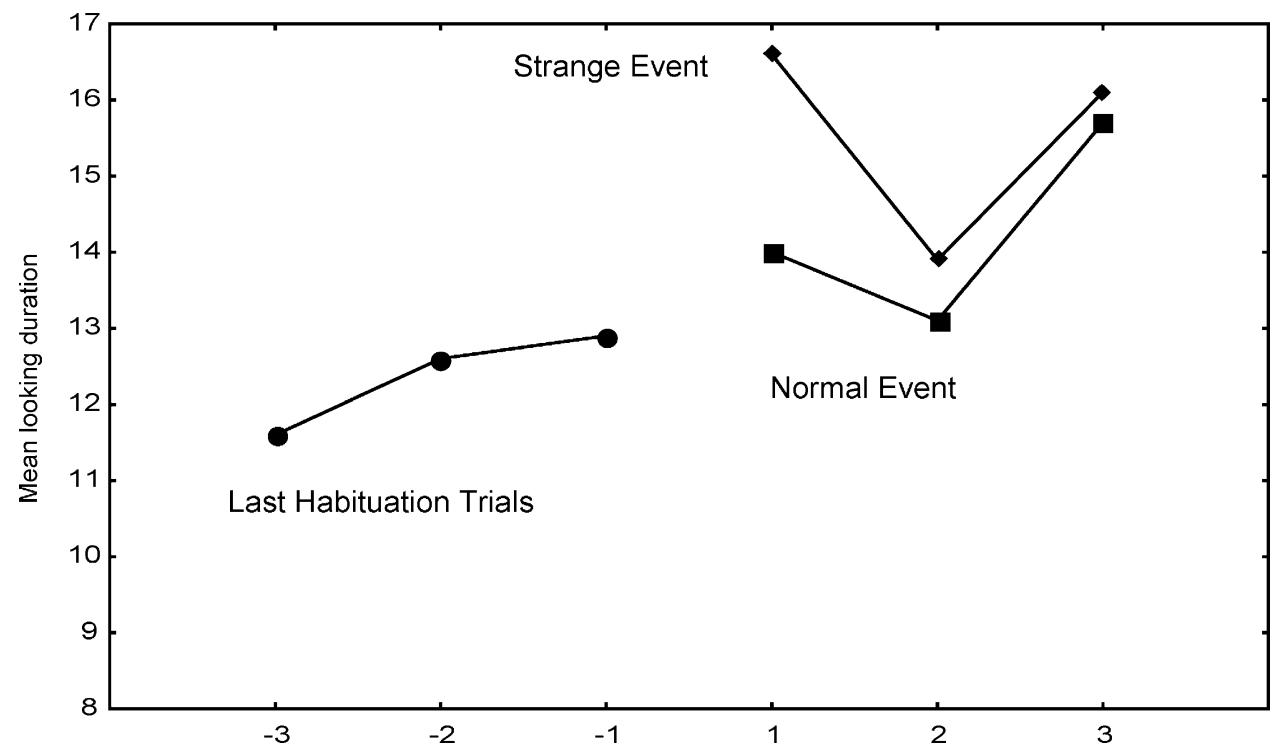

Fig. 4. Mean looking durations in seconds during the last habituation trials, normal test trials and strange test trials; experiment 2 . 
normal and the strange events. However, a novelty reaction was observed for the strange event, but not for the normal one. A possible explanation of these ambiguous results is that during the considered period, the infants learned to use depth cues in a 2-D display. Thus, more informative cues may be required to evidence greater differences in processing durations for normal and strange events. As a consequence, our second hypothesis, put forward to explain the results of the first experiment: that a sequential display of the different distances of the object is not adapted to the infants' representation of the relation between distance and size, must be tested. In fact, from trial to trial, the cube appeared each time with a new size at a new place. It may be difficult for infants to learn a rule of perspective in this condition (a sequential movement).

\section{Experiment 3}

As the sequential presentation of the perspective rule appeared to be too difficult to understand for 3-month-olds, two differences were introduced to test the same rule in the third experiment. Older infants were tested, and the same rule of perspective was presented through a continuous movement.

\subsection{Subjects}

The experiment was conducted on a sample of 36 full-term 4-month-old infants, 16 girls and 20 boys (mean age $=4$ months, 1 day). Seven additional infants were tested but could not be used as subjects because of sleeping or crying. The infants were recruited in the same way as in the previous experiments.

\subsection{Apparatus and procedure}

The apparatus used in the third experiment was the same as in the first two experiments. However, the procedure differed in many aspects. Infants were first habituated to the following situation: a curtain was drawn that showed on the TV screen a background representing a room, with perspective cues placed at the bottom, at the top and on both sides. An object was situated in the top left-hand corner of the room. This object moved along a slanted line, alternately in the direction of the vanishing point and back in the direction of its original point. When the object apparently moved away, its size decreased, and when it apparently drew near, its size increased (Fig. 5). When the infants were habituated to this situation, the curtain was closed and opened again with the object in the bottom right-hand corner. Two events, a normal and a strange one, were presented alternately. Half of the subjects saw the normal event first, the other half saw the strange event first.

In the normal event, the movement was symmetrical to that of the habituation event. As in the habituation event, the object was big when apparently near and small when apparently far, that is to say its size decreased when it moved up along a slanted line, from the corner to the vanishing point, and increased when it moved along the same line in the other direction. In the strange event, the movement was exactly the same as in the habituation phase, but simply 


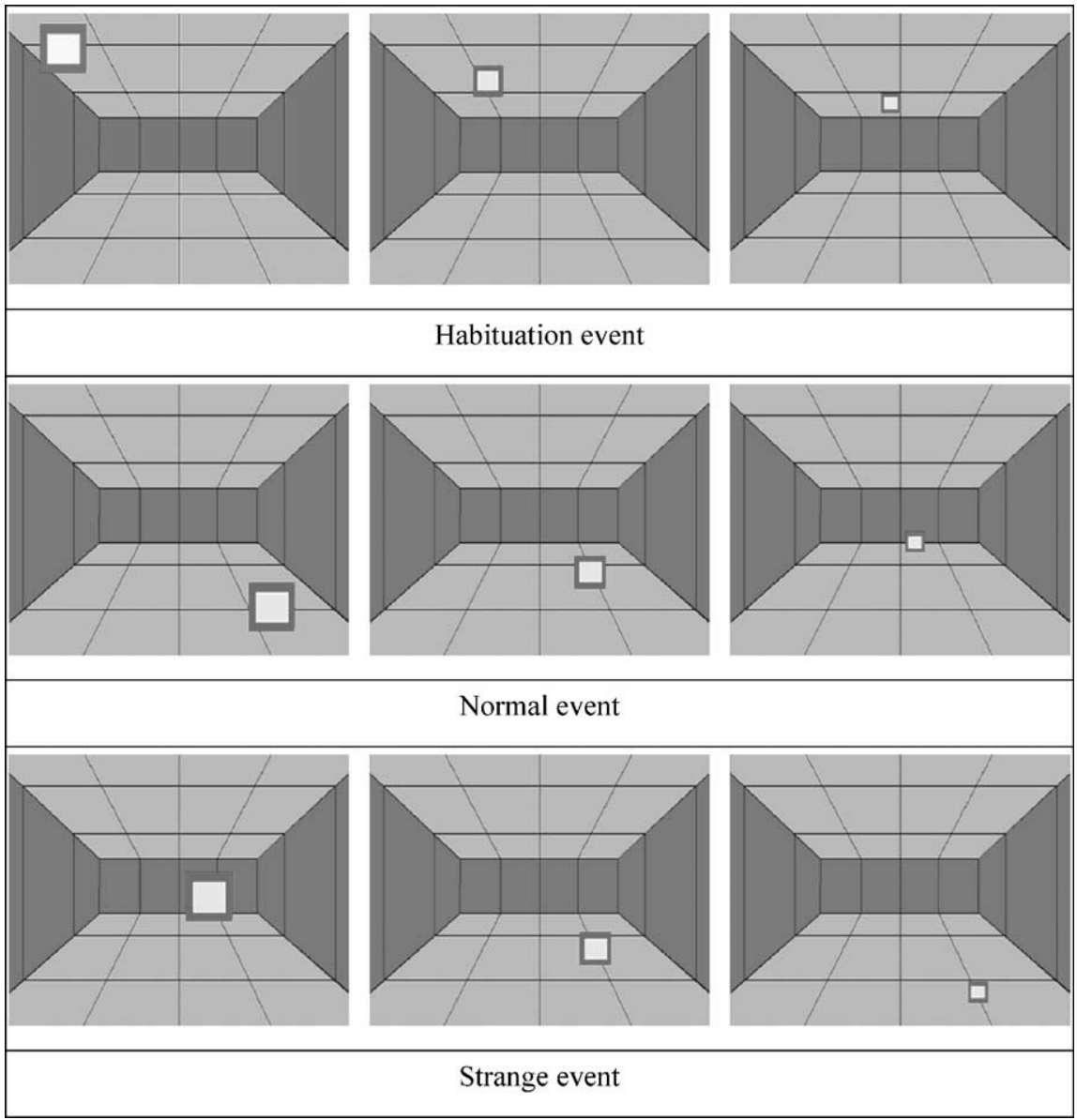

Fig. 5. The habituation events, normal and strange test events; experiment 3.

transferred forward to the bottom right hand corner. As a consequence, the object was big when apparently far and small when apparently near. This movement appeared to be very strange to adults.

If the infants were not able to use perspective cues, they would have looked longer at the normal event, which was the newer one, but if they were able to use these cues, they would have found the last event to be strange and looked at it for a longer time. Although a continuous movement situation provides clearer cues than a sequential display, since the data of our second experiment can be interpreted as the evidence of an emerging capacity, 4-month-olds were tested in this experiment.

\subsection{Results}

As shown in Fig. 6, no difference appeared in the looking durations for the normal and the strange events. Infants looked longer at the strange events but the difference was not significant 


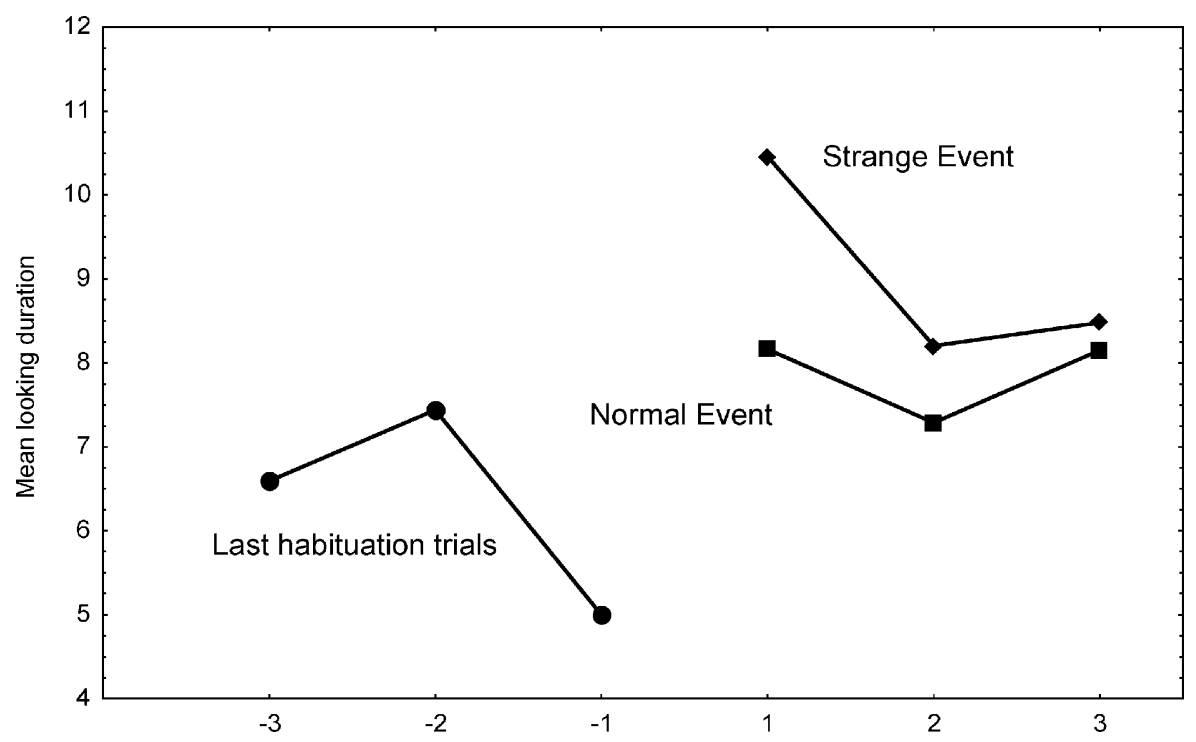

Fig. 6. Mean looking durations in seconds during the last habituation trials, normal test trials and strange test trials; experiment 3 .

$[F(1,34)=1.25, p=.272]$. Fig. 6 also indicates a significant difference between the last habituation trials and the strange event trials $[F(1,34)=8.35, p=.007]$. Such a significant novelty reaction did not appear for the normal event $[F(1,34)=2.46, p=.126]$.

\subsection{Discussion}

In spite of the differences in procedure for the second and the third experiment, the results of both appear to be very similar. No difference in the looking durations was evidenced between the normal and the strange events, but a difference was observed between the last habituation trials and the strange events. These results, again, are ambiguous but interesting because the novelty reaction appeared for the strange event, which is also the one that is less new in the test phase. However, although a continuous movement was presented to the infants in this experiment, it was not a sufficient condition to make 4-month-olds look longer at a strange event than at a normal test event.

\section{General discussion}

Three experiments were conducted in this study. In the first experiment, 3-month-olds were asked to extrapolate the rule "further away then smaller" with a sequential presentation of that rule in the habituation phase and a 2-D display. None of the results obtained indicated such an extrapolation. In the second experiment, 3-month-olds were asked to extrapolate the rule apparently nearer then bigger with the same sequential presentation of that rule in the habituation phase and the same 2-D display. No significant difference in looking duration 
was observed between the normal and the strange events, but a significant difference appeared between the last habituation trials and the strange events, and not between the end of habituation trials and the normal events. In the third experiment, a continuous movement 2-D display was used to indicate to 4-month-olds the relation between the apparent distance and size of an object. In the test phase, the same rule was presented, with the simple inversion of the movement (normal event), or the same movement as in the habituation phase was presented in an inadequate background corner (strange event). Again, no difference in looking durations appeared between the normal and the strange events, but a significant difference appeared between the last habituation trials and the strange events, not between the end of habituation trials and the normal events.

Taken altogether, the results of our three experiments do not indicate a clear mastery of the perspective rules and an unambiguous use of perspective cues with a 2-D display. However, the similitude of the results obtained in experiments 2 and 3 is interesting. In both of these cases, infants dishabituated for the less new event, but did not dishabituate for the newer event. Our results then cannot be attributed to a novelty reaction. Further, they cannot be attributed to a preference for familiarity: in both experiments, a novelty was presented in the test phase for the strange event. This dishabituation can only be explained by the fact that the strange event actually appeared to be strange to the infants. In other words, 3-month-olds (in experiment 2) and 4-month-olds (in experiment 3) seem to have used perspective cues in the 2-D display, but did not master them well enough to give more clear cut indices of this use: there was no significant difference in looking duration between the normal and the strange events.

This absence of difference in the classical index used with the present paradigm leads us back to theoretical considerations. Our results are perfectly compatible with a learning hypothesis with respect to perspective cues, and the ages tested appear to be those when this learning occurs. These results are much less compatible with the nativist theory. If this theory was true, differences in looking duration should have been evidenced between the normal and strange events in our three experiments. The observed difference between experiments 1 and 2 is especially instructive as a test of these two theoretical points of view. From a nativist point of view, no difference exists between the two situations: the logical relation presented between size and apparent distance is the same. However, from a learning point of view, the two situations are different, because the infants were more interested in nearer than in further objects.

Concerning the representational level which is necessary to explain our results, it cannot simply be our level 1: using 3-D cues in a 2-D display require the use of the same mechanisms as that used to extract the 2-D image from the 3-D stimulus, but in the opposite direction. This course corresponds to our level 2: abstract representation. Abstract representation has been defined referring essentially to categorization capacities. In fact, categorization in infants has been evidenced at the same ages as those used in our three experiments. In both of these cases, an abstraction process is going on, from the level 1 representation (translated copy) to a more abstract level, implying properties of different stimuli successively presented (categorization) or inferring properties of a never seen stimulus (passage from 2-D to 3-D).

As a conclusion of our three experiments and previous experiments in the same domain such as Lécuyer and Durand (1998), the study of 2-D representation seems to be a good paradigm for understanding early representational capacities. However, as they stand, our results can 
serve only as landmarks on the way to a better understanding of these capacities. Other 2-D displays must be presented to infants and other ages must be tested to understand how abstract representation actually emerges.

\section{References}

Baillargeon, R. (1986). Representing the existence and the location of hidden objects: Object permanence in 6- and 8-month-old infants. Cognition, 23(1), 21-41.

Baillargeon, R., \& DeVos, J. (1991). Object permanence in young infants: Further evidence. Child Development, 62(6), 1227-1246.

Baillargeon, R., \& Graber, M. (1987). Where's the rabbit? 5.5-month-old infants' representation of the height of a hidden object. Cognitive Development, 2(4), 375-392.

Baillargeon, R., Spelke, E. S., \& Wasserman, S. (1985). Object permanence in five-month-old infants. Cognition, 20(3), 191-208.

Campos, J. J., Langer, A., \& Krowitz, A. (1970). Cardiac responses on the visual cliff in prelocomotor human infants. Science, 170, 196-197.

Durand, K., \& Lécuyer, R. (2002). Object permanence observed in four-month-old-infants with a 2-D display. Infant Behavior and Development, submitted for publication.

Fagan, J. F. (1976). Infant's recognition of invariant features of faces. Child Development, 47, 627-638.

Fantz, R. L. (1961). The origin of form perception. Scientific American, 204, 66-72.

Fantz, R. L., \& Nevis, S. (1967). Pattern preference and perceptual-cognitive development in early infancy. Merrill Palmer Quarterly, 13, 77-108.

Gibson, E. J., \& Walk, R. D. (1960). The "visual cliff”. Scientific American, 202, 64-71.

Haith, M. M. (1998). Who put the cog in infant cognition? Is rich interpretation too costly? Infant Behavior and Development, 21(2), 167-179.

Hudson, W. (1960). Pictorial depth perception among sub-cultural groups in Africa. Journal of Social Psychology, $52,183-208$.

Hudson, W. (1967). The study of the problem of pictorial perception among unaccultured groups. International Journal of Psychology, 2, 89-107.

Lécuyer, R. (1994). Nouveauté et organisation des connaissances. In R. Lécuyer, M.-G. Pêcheux, \& A. Streri (Eds.), Le développement cognitif du nourrisson (Vol. 1, pp. 147-196). Paris: Nathan Université.

Lécuyer, R., \& Durand, K. (1998). Bi-dimensional representations of the third dimension and their perception by infants. Perception, 27, 465-472.

Lécuyer, R., Humbert, R., \& Findji, F. (1992). Un nouvel appareillage pour l'étude de l'habituhation chez le bébé. Année Psychologique, 92, 225-232.

Meltzoff, A. N., \& Moore, M. K. (1977). Imitation of facial and manual gestures by human neonates. Science, 198, $75-78$.

Meltzoff, A. N., \& Moore, M. K. (1998). Object representation, identity, and the paradox of early permanence: Steps toward a new framework. Infant Behavior and Development, 21(2), 201-235.

Piaget, J. (1936). La naissance de l'intelligence chez l'enfant. Neuchâtel, Paris: Delachaux et Niestlé.

Slater, A. M., Mattock, A., \& Brown, E. (1990). Size constancy at birth: Newborn infants' responses to retinal and real size. Journal of Experimental Child Psychology, 49, 314-322.

Sokolov, E. M. (1963). Perception and the conditioned reflex (first published in 1958). New York: Macmillan.

Spelke, E. S. (1998). Nativism, empiricism, and the origins of knowledge. Infant Behavior and Development, 21(2), $181-200$.

Streri, A., \& Pêcheux, M.-G. (1986). Cross-modal transfer of form in 5-month-old infants. British Journal of Developmental Psychology, 4, 161-167.

Wallon, H. (1942). De l'acte à la pensée. Paris: Flammarion.

Wynn, K. (1992). Addition and subtraction by human infants. Nature, 358, 749-750. 
Yonas, A., \& Granrud, C. E. (1985). Development of the depth sensitivity in infants. In J. Mehler \& N. Fox (Eds.), Neonate cognition: Beyond the blooming, buzzing confusion. Hillsdale, NJ: Erlbaum.

Yonas, A., \& Granrud, C. E. (1985). Reaching as a measure of infants' spatial perception. In G. Gottlieb \& N. A. Krasnegor (Eds.), Measurement of audition and vision in the first year of postnatal life: A methodological overview (pp. 301-322). Norwood, NJ: Ablex.

Yonas, A., \& Owsley, C. (1987). Development of visual space perception. In P. Salapatek \& L. Cohen (Eds.), Handbook of infant perception: From perception to cognition (Vol. 2, pp. 80-122). New York: Academic Press.

Yonas, A., Pettersen, L., \& Granrud, C. E. (1982). Infants' sensitivity to familiar size as information for distance. Child Development, 53, 1285-1290.

Yonas, A., \& Pick, H. L. (1975). An approach to the study of infant space perception. In L. B. Cohen \& P. Salapatek (Eds.), From sensation to cognition (Vol. 2). New York: Academic Press. 